Presented at the 16 th Annual Conference on Composite and Advanced Ceramics Cocoa Beach, FL, Jan 7-10 1992.

\title{
A PROCESS FOR THE FABRICATION OF CERAMIC FIBER REINFORCED
} TITANIUM ALUMINIDE

I Horsfall, S J Cundy Cranfield Institute of Technology, Shrivenham, Swindon, Wiltshire UK

This paper describes initial work on a novel process for the production of titanium aluminide matrix composites reinforced with short alumina fibers. The processing route involves an adaption of existing metal matrix composite (MMC) fabrication technology used to produce hybrid particulate/short fiber composites. A preform is produced which contains alumina fibers and titanium metal powder with a fiber content of around $10 \%$ by volume and approximately $50 \%$ porosity. This preform is then infiltrated with pure aluminum by a squeeze casting process to produce a fully dense composite of titanium powder and alumina fibers in a metallic aluminum matrix. The composite is then heat treated in a hot isostatic press to react the aluminum and titanium to produce a titanium aluminide matrix.

\section{INTRODUCTION}

Titanium aluminides have been proposed as candidate materials for gas turbine components such as compressor and turbine casings, high pressure compressors, and other medium temperature areas of the engine (1). It would be useful to be able to reinforce them with ceramic fibers. These could be expected to increase the elastic modulus, creep resistance and strength, as well as providing energy absorbing mechanisms during fracture.

It has been demonstrated that it is relatively easy to promote reaction of elemental metals either in solid or partially liquid form to produce intermetallic interfacial layers (2), or monoliths ( 3 ), where intimate contact between the constituents has been achieved by squeeze casting liquid metal into a porous preform of a second solid metal, or by cold extrusion of two elemental powders. It has been shown (4) that aluminum may be squeeze cast into an array of titania fibers and then heat treated to produce titanium aluminide. At least one attempt has been made to produce fiber reinforced intermetallics by melt infiltration (5) but their high melting point makes this a difficult process.

It has been demonstrated that hybrid MMCs incorporating fiber and particulate reinforcement may be fabricated using a squeeze casting process (6). It is possible to replace some or all of the particulate reinforcement with a metal powder such as titanium, so that a composite is produced in which the aluminum 
metal matrix contains fiber reinforcement and particles of the metal powder. Such a composite could be heat treated to totally react the titanium and aluminum without damaging the fibers so that a fiber reinforced intermetallic matrix composite was produced.

This paper describes such a process route which may be used to fabricate a titanium aluminide matrix incorporating short randomly oriented alumina fibers. The microstructures produced at each stage of the process will be described and the effect of variables in the preforming and heat treatment stages will be discussed.

\section{PREFORM MANUFACTURE}

Preforms can be fabricated in a number of mixed fiber / particulate systems. In the context of this paper SAFFIL (ICI PLC Trademark) short fibers and titanium powder were used. SAFFIL fibers are crystalline alumina with a small amount of silica to stabilise the delta alumina phase. The fibers average 3 microns in diameter and 300-500 microns in length. Preforms used one of two grades of titanium metal powder having mean sizes of $80 \mathrm{microns}$ and $20 \mathrm{microns.} \mathrm{The} \mathrm{coarser} \mathrm{powder} \mathrm{had} \mathrm{a} \mathrm{wide}$ variation in particle size and shape which proved difficult to mix with the fiber. Some preforms were made using the finer powder which produced more homogenous preforms with a much better dispersion of the fiber.

The production of the preforms follows two distinct stages. First the fiber and powder were dispersed in a suitable medium. Both organic media and aqueous media having neutral PH were used. The constituents must be dispersed so as to avoid agglomerations whilst preventing excessive fiber breakage. At this stage a binder such as latex or starch was added to the suspension to provide handling strength.

The suspension is then pressed against a filter to form a preform and pressure may be applied in order to densify the preform. With the coarser titanium powder it was found difficult to prevent some differential settling of the powder and fiber so that the top of the preform had a higher fiber content than the base. This problem was overcome with the finer powder where the degree of settling out of the metal powder was much less and there was no obvious stratification within the preform.

The quantities of titanium powder in the preform are selected according to the composition of the matrix required, although this assumes that the final matrix is homogeneous. In order to get a TiAl matrix, the final composite requires a $1: 1$ atomic ratio of aluminum to titanium in the matrix. The volumes of titanium, and the volume of porosity which will be filled with aluminum in the preform can be calculated to achieve the required contents.

The volume fraction of fiber can be varied as desired, although the maximum volume fraction is limited by the requirement for sufficient porosity to allow full infiltration during casting. Also to prevent the need for excessive compaction pressure on the 
preform which would cause fiber breakage, the fiber content is limited to approximately $30 \%$ by volume.

The preforms used in this study had titanium contents calculated to give $\mathrm{TiAl}_{3}$ as the final matrix material assuming homogeneity. The fiber content of approximately $10 \%$ was sufficiently high to prevent dense packing of the titanium powder and sufficiently low as to allow easy infiltration. This composition required that the preform had approximately 45 \% titanium by volume, which together with approximately 108 fiber left $45 \%$ porosity. The preforms were $100 \mathrm{~mm}$ in diameter and between 5 and $15 \mathrm{~mm}$ thick.

\section{INFILTRATION}

A small squeeze casting rig was used to infiltrate the preforms with liquid aluminum under pressure. All the castings used commercial purity aluminum. Although there would be advantages to using a higher purity aluminum, the lack of fluidity would make infiltration considerably more difficult. The preform was preheated to $620 \mathrm{~K}$ in the die and molten aluminum at $1270 \mathrm{~K}$ was added. A pressure of $25 \mathrm{MPa}$ was then applied by a hydraulic ram and held for 1 minute whilst the aluminum solidified. Figure 1 shows the structure of a typical composite after the casting stage and the presence of a thin reaction layer at the titanium aluminum interface can

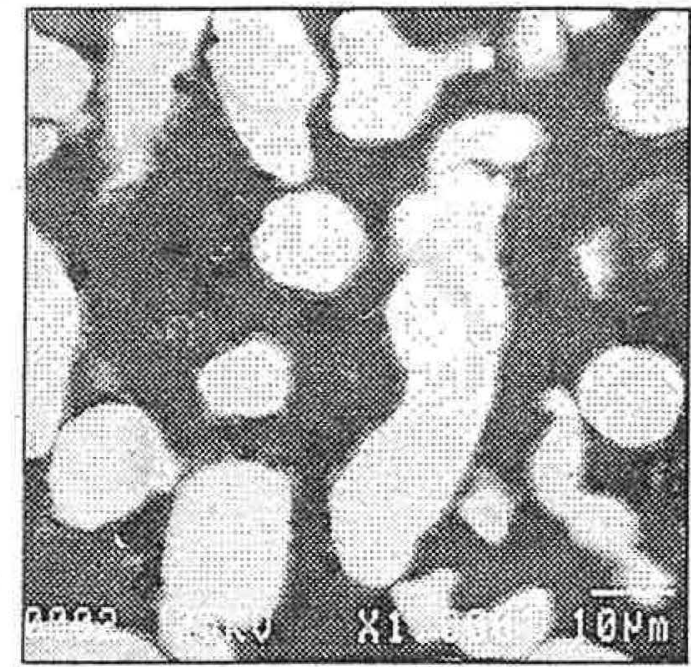

Figure 1 Backscatterred electron image (BSE) of part of cast C.in the as cast condition

be observed. This composite contained the fine titanium powder so that a good dispersion of the fiber and particulate was achieved.

\section{HEAT TREATMENT}

During the casting stage there was some limited interaction between the aluminum and titanium such that a thin reaction layer, of $\mathrm{TiAl}_{3}$ was formed. It was relatively easy to thicken the intermetaliic reaction layer, Figure 2 shows the effect of a 6 hour heat treatment at $840 \mathrm{~K}$ which had produced an intermetallic layer up to 5 microns thick at the metal interfaces. In order to get full reaction of the material it was necessary to use higher temperatures, but more severe heat treatments whilst thickening this layer also lead to the formation of Kirkendall porosity. Although a fully reacted material can be produced, there is a swelling of 20-30\% and a very porous structure is produced with a high proportion of the fibers in or adjacent to the pores. 


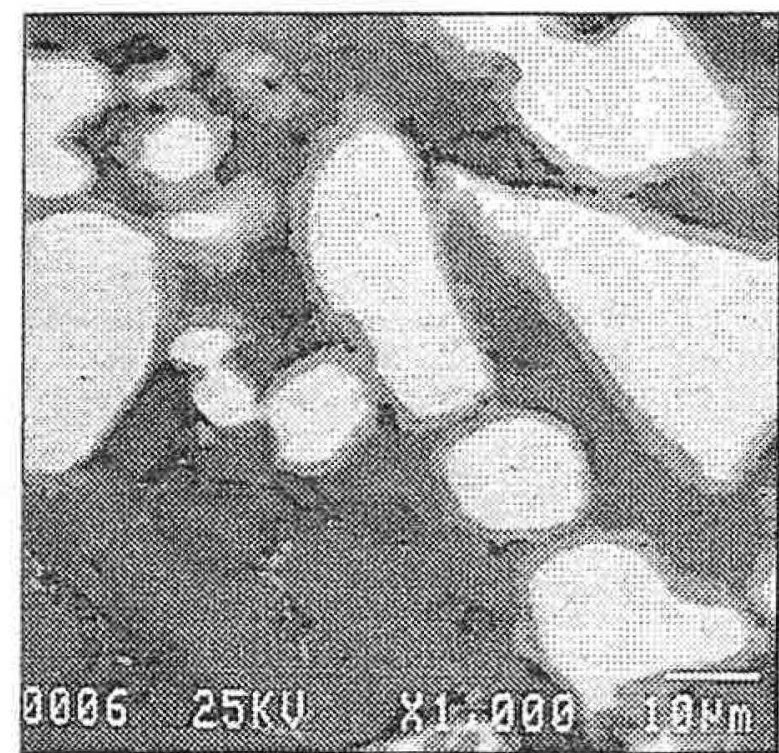

Figure 2 BSE image of

composite (Cast $C$ ) after heat treating at $873 \mathrm{~K}$ to thicken the $\mathrm{TiAl}_{3}$ layer.

In order to prevent pore formation heat treatments were carried out in a hot isostatic press (HIP) at pressures of $200 \mathrm{MPa}$.

Heat treatments have been carried out for periods of 6 hours at temperatures of 770,870 and $970 \mathrm{~K}$. Figure 3 shows the structure produced after heat treatment at $870 \mathrm{~K}$ for 6 hours. It can be seen that the titanium and aluminum have been almost totally reacted to form compounds.

Table 1

\begin{tabular}{|c|c|c|c|c|}
\hline $\begin{array}{l}\text { Cas } \\
t\end{array}$ & $\begin{array}{l}\text { Matrix Ti } \\
\text { content } \\
\text { Atomic } \%\end{array}$ & $\begin{array}{l}\text { Fiber } \\
\text { content } \\
\text { Volume } 8\end{array}$ & $\begin{array}{l}\text { Ti } \\
\text { particle } \\
\text { size } \\
\text { microns }\end{array}$ & $\begin{array}{l}\text { HIP } \\
\text { temperatu } \\
\text { re } \\
\mathrm{K} \\
\end{array}$ \\
\hline $\mathrm{A}$ & 50 & 10 & 80 & 770 \\
\hline B & 50 & 10 & 80 & 870 \\
\hline C & 40 & 8 & 20 & 970 \\
\hline
\end{tabular}

Table 1 summarises the composition and heat treatment schedules used to produce composite material.

\section{METALLOGRAPHY}

Specimens were sectioned and polished after heat treatments in order to examine the structures produced. Energy dispersive $x$-ray analysis was used to establish the composition of the various phases present. This analysis has been used as the basis for determining which titanium aluminide phase is formed.

Cast A showed only moderate levels of reaction with a 5-7 micron $\mathrm{TiAl}_{3}$ layer around the titaniumparticles. The composite integrity was retained with no obvious pore formation and the 


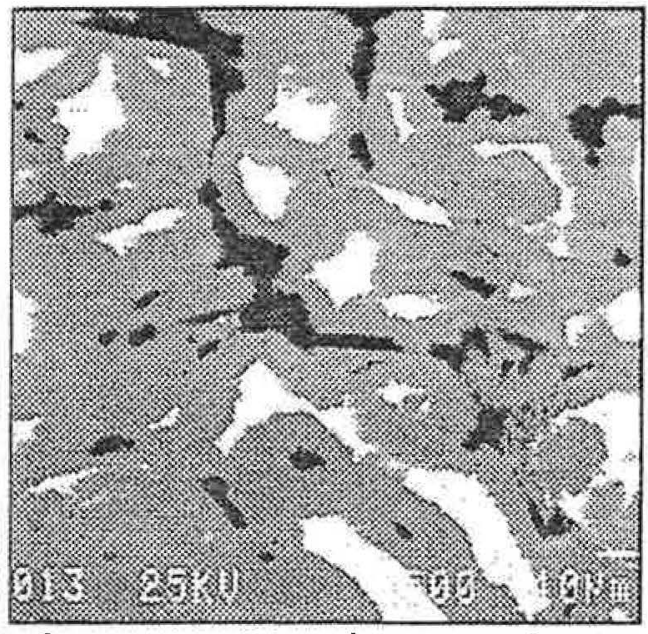

Figure 4 BSE image of the same sample as fig 3 showing an area in which unreacted $\mathrm{Ti}$ (bright phase) is still present.

fibers showed no sign of damage. The alumina fibers were transparent under optical examination, the continued transparency suggesting that there was no gross chemical attack or grain growth during the heat treatment. After heat treatment the specimens showed a decrease in volume of between 0.63 and 0.86 \& which can be attributed to the closing up of small internal pores.

Cast $B$ used a higher temperature to produce a more complete reaction. The results of this heat treatment schedule were somewhat variable. Some parts of the specimen material showed complete reaction to $\mathrm{TiAl}_{3}$ with good homogeneity (Figure 3 ). There were also areas which showed unreacted titanium and aluminum (Figure 4), and large parts of the specimen were porous. It was determined that the HIP treatment had reached $870 \mathrm{~K}$ sometime before the pressure had accumulated resulting in the formation of pores in parts of the specimen and that the heat treatment was either too short or at too low a temperature to allow complete reaction.

For cast $\mathrm{C}$ a finer titanium particle size was used and the heat treatment temperature was increased in order to attain a uniform greater degree of reaction. Care was also taken to delay heating until the HIP had reached full pressure. An even reaction was produced over the whole specimen but although all the titanium was reacted there was still an amount of remaining unreacted aluminum. There was also evidence of extensive degradation of the fibers which had a grainy and broken up appearance.

A sample of the heat treated material from cast $C$ was annealed for 3 hours at $1520 \mathrm{~K}$ in air. There was no obvious modification to the aluminide phase or the fiber remains, but some large precipitates of composition Ti-15Al-5Si appeared (Figure 5). The silicon content had probably been extracted from the fibers which contain a small amount of silica, and thus tends to indicate that the fiber attack is due to the extraction of silica from the delta alumina with subsequent breakdown of the fiber.

\section{DISCUSSION}

The short exposure to liquid aluminum that the titanium experiences during casting leads to the formation of a thin even reaction layer around the particles. This can be thickened by mild heat treatment(Figure 4 ) or by Hipping. At $770 \mathrm{k}$ it appears possible to get extensive reaction such that a suitably fine titanium powder could be totally reacted to obtain an aluminide. In all cases the reaction layer shows sharp boundaries towards each metal interface and no concentration gradient is observed. Energy dispersive $\mathrm{x}$-ray analysis showed the reaction layer under all the heat treatments to contain 
atomic percentages of approximately 218 titanium and 798 aluminum which suggests an aluminum rich $\mathrm{TiAl}_{3}$ as the main phase. Even in areas where all the aluminum had been reacted and some unreacted titanium remained the composition remained similar. This is in agreement with work by Loo and Reideck (7) which showed the formation of $\mathrm{TiAl}_{3}$ in titanium aluminum diffusion couples to the exclusion of all other phases due to the high mobility of aluminum in this phase. It has been shown (3) that TiAl and $\mathrm{Ti}_{3} \mathrm{Al}$ may be formed by the reaction of elemental powders but this requires higher temperatures and titanium contents. The annealed sample from cast $C$ showed the presence of a titanium aluminide of a composition equal to $\mathrm{Ti}_{3} \mathrm{Al}$ but with

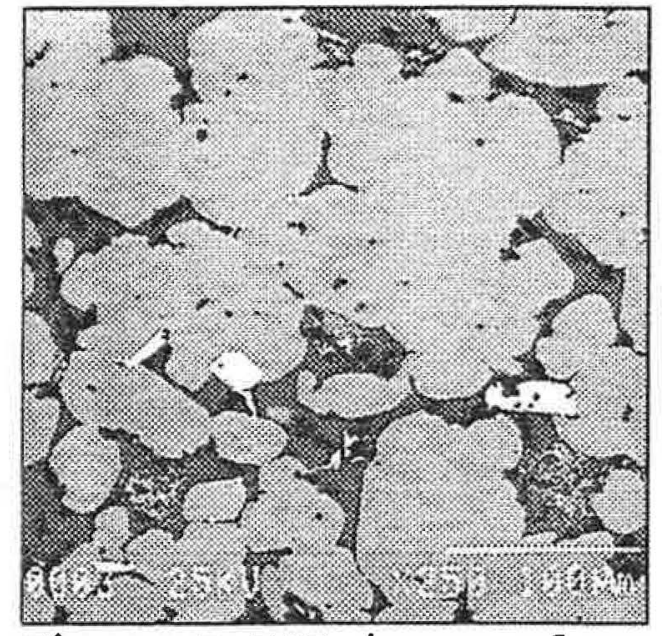

Figure 5 BSE image of an annealed sample from cast $C$, showing the formation of Ti-Al-Si particles (bright phase) some silicon present. Therefore it does seem possible that a titanium rich matrix could be formed given the correct starting composition and heat treatment.

\section{CONCLUSION}

It would appear that with minor changes to the process conditions using the fine titanium powder and hipping at $770 \mathrm{~K}$ a homogenous $\mathrm{TiAl}_{3}$ matrix containing undamaged fibers may be produced. It has been suggested (8) that $\mathrm{TiAl}_{3}$ may be a suitable material for high temperature applications particularly if modified by the addition of transition elements to produce the $\mathrm{L}_{1}$ structure which would reduce the room temperature brittleness. With higher titanium compositions it should be possible to produce a matrix containing more of the titanium rich phases although it is not clear whether the higher processing conditions will degrade the fiber. Cast $C$ was hipped at above the melting point of the aluminum phase so that the fibers where exposed to liquid aluminum for several hours. It may be that a lower initial heat treatment temperature followed by a higher temperature anneal without allowing melting might reduce the fiber damage. Alternatively another fiber type could be used. It has been reported (9) that FP alumina fibers have survived heat treatments of $1533 \mathrm{~K}$ in $\mathrm{Ni}_{3} \mathrm{Al}$ matrices without damage although this may be due to the absence of a liquid phase.

During future work it is hoped that the processing conditions for a short fiber titanium aluminide composite can be optimised. The process could then be developeed to include continuous fibers and other binary and multicomponent matrix systems.

\section{ACKNOWLEDGEMENTS}

The authors wish to thank Dr David Stephenson of Cranfield Institute of Technology Bedford, for the hot isostatic pressing of the material used in this work and to ICI Chemicals and 
Polymers for fibers.

\section{REFERENCES}

1) H. A. Lipsitt, "Titanium aluminides-future turbine materials", Proc. Advanced high temperature alloys processing and properties, Cambridge Mass 16-18 June (1985).

2) S. Nagato and M Sakamoto, "Development and applications of metal composites from pressure casting", Materials and Design, 10 No 3 (1989) 153-158.

3)M. Dahms, F. Schmelzer, J Seeger, and B. Wildhagen, "Microstructure and mechanical properties of a gamma-base titanium aluminide produced from extruded elemental powders", Proc High Temperature Intermetallics, 30 April-1 May, London, (1991) 112-121.

4) H. Fukunaga, X. Wang, and Y. J. Aramaki, "Preparation of intermetaliic compound matrix composites by reaction squeeze casting", Mat Sci Letters, 9 (1990) 23-25.

5) S. Nourbaksh, "Fabrication of nickel aluminide composites", Materials and processing report, (MIT) 3 (1989).

6) C. M. Friend, I. Horsfall, and C. L. Burrows, "The effect of particulate:fiber ratio on the properties of short fiber/particulate hybrid MMC produced by preform infiltration", J. Mat. Sci. 26 (1990) 225-231.

7) F. J. J. van Loo, and G. D. Reick, "Diffusion in the titanium-aluminum system - 1. Interdiffusion between solid Al and Ti of Ti-Al alloys", Acta Met 21 (1973), 51-71.

8) D.G. Morris, and R. Lerf, "Deformation and ductility in alloys based on TiAl ${ }_{3}$, Proc High Temperature Intermetallics, 30 April-1 May, London, (1991) 213-215.

9) C. Lee D. S. Grummon, and G. Gottstein, "Microstructure and interface behaviour in diffusion bonded $\mathrm{Ni}_{3} \mathrm{Al}+\mathrm{B}$ matrices containing continuous $\mathrm{Al}_{2} \mathrm{O}_{3}$ fibers", Proc' High Temperature Intermetalics, 30 April-1 May, London, (1991) 210-212. 